\title{
Corrigendum to: Multi-period experimental asset markets with distinct fundamental value regimes
}

\author{
Thomas Stöckl $^{1} \cdot$ Jürgen Huber $^{1} \cdot$ Michael Kirchler $^{1,2}$
}

Published online: 9 May 2015

(C) Economic Science Association 2015

\section{Corrigendum to: Exp Econ (2015) 18:314-334 DOI 10.1007/s10683-014-9404-1}

In the original version of the paper (currently available online first) we report incorrect values of RAD, RD, SPREAD, VOLA, and ST for one out of five treatments, namely $\mathrm{R} 5(\mathcal{N})$. In this corrigendum we present the corrected values in the respective tables and detail changes in the reported results. Note that we only update those tables containing incorrect values and that only values concerning $\mathrm{R} 5(\mathcal{N})$ are subject to changes. Overall, the values of RAD and RD reveal that markets are more efficient than previously reported, while values of SPREAD, VOLA, and ST are close to the values previously reported. The changes necessitate small correction in the results section listed in Table $1 .{ }^{1}$

\footnotetext{
1 We thank Owen Powell for pointing us at the mistakenly reported values.
}

The online version of the original article can be found under doi:10.1007/s10683-014-9404-1.

Thomas Stöckl

thomas.stoeckl@uibk.ac.at

Jürgen Huber

juergen.huber@uibk.ac.at

Michael Kirchler

michael.kirchler@uibk.ac.at

1 Department of Banking and Finance, Innsbruck University School of Management, Universitätsstrasse 15, 6020 Innsbruck, Austria

2 Department of Economics, Centre for Finance, University of Gothenburg, P.O. Box 600, 40530 Gothenburg, Sweden 
Table 1 Corrections, highlighted in bold face, in the results sections

Original paper Correction

\section{Third paragraph}

In particular, RAD (mispricing) with 43.2 percent is significantly higher in $\mathrm{R} 1(\mathrm{l})$ than in regimes $\mathrm{R} 2(-), \mathrm{R} 3(/)$, and R4(tri)

\section{Fifth paragraph}

In particular, in $\mathrm{R} 4($ tri) and $\mathrm{R} 5(\mathcal{N})$ mispricing is at an intermediate level between $\mathrm{R} 1(\mathrm{)})$ and $\mathrm{R} 2(-)$ with values between 8.1 and 16.5 percent for the aggregate of paths $A$ and $B$. In markets with path $\mathrm{A}, \mathrm{FV}$ s are on average higher than the unconditional expected value of 45 Taler and undervaluation equals 5.8 percent $(\mathrm{R} 4($ tri $))$ and 3.6 percent $(\mathrm{R} 5(\mathcal{N}))$, respectively. The opposite holds for markets with path B where FVs are on average lower than the unconditional expected value. These markets exhibit overvaluation of 11.5 and 15.8 percent for R4(tri) and R5 $(\mathcal{N})$, respectively

\section{Sixth paragraph}

Average values for $S T$ range from 2.63 to 3.83 for different regimes
In particular, RAD (mispricing) with 43.2 percent is significantly higher in $\mathrm{R} 1(\mathrm{l})$ than in each of the other regimes

In particular, in $\mathrm{R} 4($ tri) and $\mathrm{R} 5(\mathcal{N})$ mispricing is at an intermediate level between R1( $)$ and R2(一) with values between 8.1 and $\mathbf{1 5 . 8}$ percent for the aggregate of paths A and B. In markets with path $\mathrm{A}, \mathrm{FVs}$ are on average higher than the unconditional expected value of 45 Taler and undervaluation equals 5.8 percent (R4(tri)) and 3.1 percent $(\mathrm{R} 5(\mathcal{N}))$, respectively. The opposite holds for markets with path B where FVs are on average lower than the unconditional expected value. These markets exhibit overvaluation of 11.5 and $\mathbf{1 5 . 1}$ percent for $\mathrm{R} 4($ tri) and $\mathrm{R} 5(\mathcal{N})$, respectively

Average values for $S T$ range from 2.63 to $\mathbf{3 . 9 8}$ for different regimes

Table 2 Regime averages for RAD, RD, SPREAD, VOLA and ST

\begin{tabular}{llrrrrr}
\hline Regime & Path & RAD $(\%)$ & RD $(\%)$ & SPREAD $(\%)$ & VOLA (\%) & ST \\
\hline R1() & & 43.2 & 39.9 & 16.6 & 9.7 & 3.67 \\
R2(一) & & 1.5 & 1.3 & 2.1 & 1.4 & 2.63 \\
R3(/) & & 15.9 & -15.2 & 5.1 & 2.8 & 2.86 \\
R4(tri) & Path A & 8.1 & -5.8 & 2.3 & 1.2 & 2.48 \\
& Path B & 11.7 & 11.5 & 6.8 & 2.7 & 4.08 \\
R5(N) & Path A & 7.8 & -3.1 & 10.0 & 5.7 & 3.68 \\
& Path B & 15.8 & 15.1 & 6.2 & 2.6 & 3.98 \\
\hline
\end{tabular}


Table 3 Differences between regime averages (column minus row) in percentage points for RAD, RD, SPREAD, VOLA, and ST

\begin{tabular}{|c|c|c|c|c|}
\hline RAD & $\mathrm{R} 2(-)$ & $\mathrm{R} 3(/)$ & R4(tri) & $\mathrm{R} 5(\mathcal{N})$ \\
\hline $\mathrm{R} 1(\backslash)$ & $-41.7 \% * * *$ & $-27.3 \% * *$ & $-33.3 \% * *$ & $-31.4 \% * *$ \\
\hline $\mathrm{R} 2(-)$ & & $14.4 \% * * *$ & $8.4 \% * * *$ & $10.3 \% * * *$ \\
\hline $\mathrm{R} 3(/)$ & & & $-5.9 \%$ & $-4.1 \%$ \\
\hline R4(tri) & & & & $1.9 \%$ \\
\hline RD & $\mathrm{R} 2(-)$ & $\mathrm{R} 3(/)$ & R4(tri) & $\operatorname{R5}(\mathcal{N})$ \\
\hline $\mathrm{R} 1(\backslash)$ & $-38.6 \% * * *$ & $-55.0 \% * * *$ & $-37.0 \% * *$ & $-33.9 \% *$ \\
\hline $\mathrm{R} 2(-)$ & & $-16.5 \% * * *$ & $1.5 \%$ & $4.7 \%$ \\
\hline $\mathrm{R} 3(/)$ & & & $18.0 * *$ & $21.2^{*}$ \\
\hline R4(tri) & & & & 3.2 \\
\hline SPREAD & $\mathrm{R} 2(-)$ & $\mathrm{R} 3(/)$ & $\mathrm{R} 4$ (tri) & $\mathrm{R} 5(\mathcal{N})$ \\
\hline $\mathrm{R} 1(\backslash)$ & $-14.5 \% * * *$ & $-11.5 \% * * *$ & $-12.1 \% * * *$ & $-8.5 \% *$ \\
\hline $\mathrm{R} 2(-)$ & & $3.0 \% * *$ & $2.4 \% *$ & $6.0 \% * *$ \\
\hline $\mathrm{R} 3(/)$ & & & $-0.6 \%$ & $3.0 \%$ \\
\hline R4(tri) & & & & $3.6 \% *$ \\
\hline VOLA & $\mathrm{R} 2(-)$ & $\mathrm{R} 3(/)$ & R4(tri) & $\mathrm{R} 5(\mathcal{N})$ \\
\hline $\mathrm{R} 1(\backslash)$ & $-8.3 \% * * *$ & $-6.9 \% * *$ & $-7.7 \% * * *$ & $-5.5 \% * *$ \\
\hline $\mathrm{R} 2(-)$ & & $1.4 \% *$ & $0.6 \%$ & $2.8 \% * *$ \\
\hline $\mathrm{R} 3(/)$ & & & $-0.9 \%$ & $1.4 \%$ \\
\hline R4(tri) & & & & $2.0 \% *$ \\
\hline ST & $\mathrm{R} 2(-)$ & $\mathrm{R} 3(/)$ & R4(tri) & $\mathrm{R} 5(\mathcal{N})$ \\
\hline $\mathrm{R} 1(\backslash)$ & -1.04 & -0.81 & -0.39 & 0.16 \\
\hline $\mathrm{R} 2(-)$ & & 0.23 & 0.65 & 1.21 \\
\hline $\mathrm{R} 3(/)$ & & & 0.42 & 0.97 \\
\hline R4(tri) & & & & 0.55 \\
\hline
\end{tabular}

$*, * *, * * *$ and denote the $10 \%, 5 \%$ and $1 \%$ significance levels, derived from double-sided MannWhitney U-tests 
Table 5 Individual market results for RAD, RD, SPREAD, VOLA and ST for regimes R1( ) to R5 $(\mathcal{N})$

\begin{tabular}{|c|c|c|c|c|c|c|}
\hline Regime & Market & RAD (\%) & $\mathrm{RD}(\%)$ & SPREAD $(\%)$ & VOLA (\%) & ST \\
\hline \multirow[t]{6}{*}{ R1(〉) } & 1 & 25.6 & 16.7 & 16.2 & 16.1 & 2.08 \\
\hline & 2 & 44.7 & 44.4 & 15.9 & 11.2 & 5.79 \\
\hline & 3 & 54.9 & 54.5 & 36.0 & 5.5 & 3.28 \\
\hline & 4 & 8.5 & 1.2 & 8.0 & 5.0 & 3.36 \\
\hline & 5 & 65.9 & 62.9 & 13.4 & 7.8 & 3.65 \\
\hline & 6 & 59.4 & 59.4 & 10.3 & 12.6 & 3.87 \\
\hline Mean & & 43.2 & 39.9 & 16.6 & 9.7 & 3.67 \\
\hline \multirow[t]{6}{*}{$\mathrm{R} 2(-)$} & 1 & 0.1 & 0.0 & 0.4 & 0.2 & 2.60 \\
\hline & 2 & 0.9 & 0.6 & 5.1 & 4.6 & 3.04 \\
\hline & 3 & 0.9 & 0.2 & 2.7 & 1.6 & 1.30 \\
\hline & 4 & 5.9 & 5.9 & 1.4 & 0.6 & 2.13 \\
\hline & 5 & 0.5 & 0.5 & 1.5 & 0.7 & 4.30 \\
\hline & 6 & 0.7 & 0.7 & 1.6 & 0.8 & 2.41 \\
\hline Mean & & 1.5 & 1.3 & 2.1 & 1.4 & 2.63 \\
\hline \multirow[t]{6}{*}{$\mathrm{R} 3(/)$} & 1 & 7.1 & -5.6 & 5.1 & 2.6 & 2.01 \\
\hline & 2 & 16.2 & -15.2 & 3.9 & 1.5 & 2.32 \\
\hline & 3 & 1.1 & -0.6 & 2.2 & 1.5 & 4.70 \\
\hline & 4 & 22.6 & -22.3 & 4.8 & 1.8 & 1.20 \\
\hline & 5 & 28.0 & -28.0 & 9.1 & 7.0 & 3.57 \\
\hline & 6 & 20.1 & -19.2 & 5.7 & 2.5 & 3.40 \\
\hline Mean & & 15.9 & -15.2 & 5.1 & 2.8 & 2.86 \\
\hline \multirow[t]{3}{*}{ R4(tri) } & $1(\mathrm{~A})$ & 7.2 & -6.8 & 1.5 & 1.0 & 3.53 \\
\hline & $3(\mathrm{~A})$ & 5.0 & 1.3 & 2.0 & 0.9 & 1.88 \\
\hline & $5(\mathrm{~A})$ & 12.0 & -12.0 & 3.3 & 1.7 & 2.05 \\
\hline \multirow[t]{4}{*}{ Mean } & & 8.1 & -5.8 & 2.3 & 1.2 & 2.48 \\
\hline & 2 (B) & 14.1 & 13.7 & 7.2 & 3.7 & 7.94 \\
\hline & 4 (B) & 9.4 & 8.9 & 3.1 & 1.5 & 2.29 \\
\hline & 6 (B) & 11.7 & 11.7 & 10.1 & 2.9 & 2.01 \\
\hline Mean & & 11.7 & 11.5 & 6.8 & 2.7 & 4.08 \\
\hline \multirow[t]{3}{*}{$\mathrm{R} 5(\mathcal{N})$} & 1 (A) & 6.2 & -2.9 & 4.5 & 2.5 & 4.78 \\
\hline & $3(\mathrm{~A})$ & 8.4 & -7.8 & 8.3 & 4.6 & 1.44 \\
\hline & $5(\mathrm{~A})$ & 9.0 & 1.4 & 17.2 & 10.0 & 4.81 \\
\hline \multirow[t]{4}{*}{ Mean } & & 7.8 & -3.1 & 10.0 & 5.7 & 3.68 \\
\hline & 2 (B) & 18.9 & 18.9 & 10.2 & 3.7 & 2.80 \\
\hline & 4 (B) & 20.1 & 20.1 & 4.5 & 2.0 & 6.15 \\
\hline & $6(\mathrm{~B})$ & 8.3 & 6.2 & 3.9 & 2.2 & 3.00 \\
\hline Mean & & 15.8 & 15.1 & 6.2 & 2.6 & 3.98 \\
\hline
\end{tabular}

